\title{
Nonlinear Stress Propagation in the Earth's Upper Mantle
}

\author{
H. J. Melosh \\ Division of Geological and Planetary Sciences, California Institute of Technology. Pasadena, California 9II25
}

\begin{abstract}
This paper consists of two parts. The first is theoretical and extends Elsasser's theory of stress propagation in the upper mantle to an asthenosphere with nonlinear rheology. Exact solutions of the nonlinear equations are found for two geologically important problems. The second part uses these theoretical results as the basis for a measurement of the rheology of the asthenosphere. The seaward migration pattern of aftershocks from the February 4, 1965, Rat Island earthquake is analyzed, and strong evidence for a non-Newtonian stress-strain relation in the asthenosphere is presented. It is found that an individual large earthquake can influence the regional stress pattern only to a distance of about $300 \mathrm{~km}$ perpendicular to the line of rupture. Excellent agreement is found between the stress propagation coefficient calculated from the aftershock migration pattern and that calculated from laboratory measurements of high-temperature creep in olivine. We thus arrive at a picture of stress propagation in the upper mantle which is consistent both with theoretical expectation and with observational evidence.
\end{abstract}

\section{INTRODUCTION}

In 1967, Elsasser [1967] propounded a theory of stress propagation in an elastic lithosphere overlying a viscous asthenosphere. Elsasser showed that provided both the lithosphere and the asthenosphere are thin in comparison to the breadth of the stress distribution, the displacement of the lithosphere from an unstressed state is described by a diffusion-type equation. $\mathrm{Re}$ cent work on the migration of earthquake epicenters [Anderson, 1975] has used Elsasser's theory, and it seems likely that this type of theory is well adapted to the description of stresses in lithospheric plates.

Recent progress in understanding high-temperature creep of rock [Weertman and Weertman, 1975] has, however, led to doubt about the validity of assuming that the asthenosphere exhibits Newtonian viscosity. It seems far more likely that the stress-strain relation in the asthenosphere is nonlinear, of the form

$$
\dot{\epsilon}_{i j}=A \sigma^{n-1} \sigma_{i j}
$$

where $\dot{\epsilon}_{\imath,}$ is the strain rate tensor, $A$ is a dimensional parameter (which is an exponential function of the absolute temperature), $\sigma_{\iota \jmath}$ is the deviatoric stress tensor, and $\sigma=\left[\frac{1}{2} \operatorname{Tr}\left(\sigma_{\ell \jmath} \sigma_{j k}\right)\right]^{1 / 2}$ is the second invariant of $\sigma_{i j}$. The value of $n$ for possible mantle materials is uncertain but probably lies between 2 and 6 [Weertman and Weertman, 1975].

If the rheology of the asthenosphere is nonlinear $(n \neq 1)$, then the Elsasser theory must be modified. Such modification is the subject of the first part of this paper. The nonlinear equations of motion are derived in the first section. Subsequent sections show that exact solutions of these equations may be obtained in two cases of geologic interest. The first case is that of a sudden change in position of the edge of a lithospheric plate, approximating conditions in the oceanic plate following a decoupling earthquake. The second case describes the displacements in a plate whose edge suddenly begins to move with constant velocity, approximating a change in forces acting on the edge of a lithospheric plate, or the average effect of a large number of decoupling earthquakes.

The pattern of displacement and stress propagation following a decoupling earthquake is found to be very sensitive to the rheology of the asthenosphere. Such sensitivity allows a direct measurement of the asthenosphere's rheology, one which is

Copyright $@ 1976$ by the American Geophysical Union. not very dependent upon model assumptions. Ideally, such a measurement requires geodetic information on the displacement of the oceanic plate following a large decoupling earthquake. Displacements should be measured at distances out to about $500 \mathrm{~km}$ from the edge of the plate and at time intervals of a few days to tens of years after the earthquake. Such information is not available for any large earthquake and is unlikely to be available in the near future. As a makeshift, we study the migration of aftershocks into the oceanic plate following a large decoupling earthquake (the February 4, 1965, Rat Island event). This approach involves the supposition that the aftershock pattern accurately delineates the pattern of stress in the lithosphere and that stress relief due to the aftershocks themselves does not significantly alter the regional pattern.

In spite of the crudeness of this approach, the aftershock migration pattern of the 1965 Rat Island event clearly shows that the rheology of the asthenosphere is not linear. The precise value of $n$ is more uncertain; any $n$ between 2 and 7 can fit the data. The main conclusion of this study is thus the simple fact that the asthenosphere is nonlinear.

This result is generally in line with the findings of Post and Griggs [1973], who studied the Fennoscandian postglacial rebound. The work of Post and Griggs, however, is crucially dependent upon a scaling assumption of unproven validity. The present work is far less dependent upon such model assumptions. In particular, the nonlinearity is handled exactly, and we have no need of hypothetical scaling laws.

\section{Part I: The Theory of Nonlinear Stress Propagation}

\section{Fundamental Equations}

Following Elsasser [1967], we let $\sigma_{x}(x)$ be the stress along the $\hat{x}$ direction in a lithospheric plate of thickness $h_{1}$ and Young's modulus $E$ (see Figure 1). We suppose this plate to be of infinite (or semi-infinite) horizontal extent and to be of negligible thickness in comparison to the distance over which $\sigma_{x}$ varies significantly (we shall see that these are reasonable approximations for several cases of geologic interest). The net force per unit area acting on the plate is $F=h_{1}\left(\partial \sigma_{x} / \partial x\right)$. This force is balanced by drag forces in the asthenosphere, $\sigma_{x z}$ per unit area, where $\sigma_{x z}$ is the shear stress at the top of the asthenosphere. In an elastic material $\sigma_{x}=E\left(\partial u_{x} / \partial x\right)$, where $u_{x}$ is the displacement of the lithosphere in the $\hat{x}$ direction. Thus 


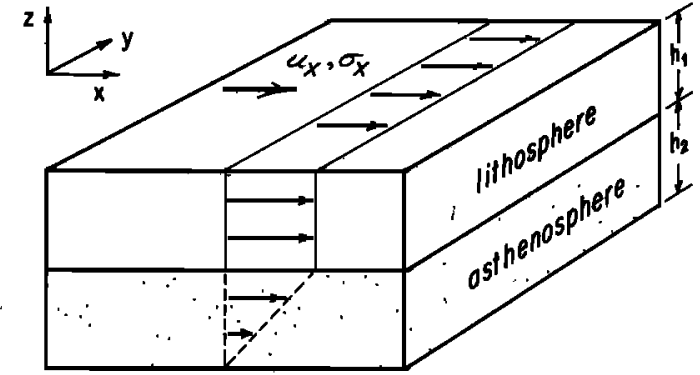

Fig. 1. Definition of various quantities and the velocity distribution $\partial u_{x} / \partial t$ in the upper mantle [after Elsasser, 1967].

$$
\sigma_{x z}=h_{1} \frac{\partial \sigma_{x}}{\partial x}=h_{1} E \frac{\partial^{2} u_{x}}{\partial x^{2}}
$$

The shear stress $\sigma_{x z}$ across the asthenosphere is independent of depth $z$ in the thin asthenosphere approximation. This result is readily deduced from the equation of equilibrium

$$
\frac{\partial \sigma_{x x}}{\partial x}+\frac{\partial \sigma_{x z}}{\partial z}=0
$$

The difference in $\sigma_{x z}$ between the top and the bottom of the asthenosphere is roughly $\Delta \sigma_{x z}=-\left(\partial \sigma_{x x} / \partial x\right) \Delta z$. For a given horizontal distribution of stress, $\partial \sigma_{x x} / \partial x$ is fixed. Thus $\Delta z \rightarrow 0$ implies $\Delta \sigma_{x z} \rightarrow 0$, proving that when the asthenosphere is thin in comparison to the horizontal distance over which stresses vary significantly, $\sigma_{x z}$ becomes independent of depth.

If $\sigma_{x z}$ is independent of $z$, then so is the right-hand side of (1); hence $\dot{\epsilon}_{x z}=$ const. But $\dot{\epsilon}_{x z}=\frac{1}{2}\left(\partial \dot{u}_{x} / \partial z\right)$, which implies that the velocity $\dot{u}_{x}$ is a linear function of depth $z$ for any power $n$ in the thin asthenosphere approximation. This situation is illustrated in Figure 1.

Equation (1) shows that the velocity of the top of the asthenosphere relative to the deep mantle is

$$
\frac{\partial u_{x}}{\partial t}=2 A h_{2}\left|\sigma_{x z}\right|^{n-1} \sigma_{x z}
$$

where $h_{2}$ is the thickness of the asthenosphere. Combining (2) and (4), we arrive at the fundamental equation for stress propagation

$$
\frac{\partial u_{x}}{\partial t}=\kappa\left|\frac{\partial^{2} u_{x}}{\partial x^{2}}\right|^{n-1} \frac{\partial^{2} u_{x}}{\partial x^{2}}
$$

where $\kappa$ is the propagation coefficient

$$
\kappa=2 A h_{2}\left(h_{1} E\right)^{n}
$$

Note that (5) and (6) reduce to Elsasser's equations if we set $n$ $=1$ and $A=1 / 2 \eta$.

Equation (5) describes the motion of the displacement field in a lithospheric plate, providing the displacements are in the $\hat{x}$ direction and are independent of $y$. If the displacements are parallel to the $\hat{y}$ axis but are still constant along $\hat{y}$ (analogous to strike-slip motion on a long fault), the equation for $u_{y}$ is of the same type as (5), only instead of using Young's modulus in $\kappa$, the rigidity $\mu$ of the lithosphere must be used. Vertical displacements $u_{z}$ cannot be described by an equation of the same type as (5), and we shall not consider them in this paper. (Vertical displacements obey an equation of form $\partial u_{z} / \partial t=$ $K\left|\partial u_{z} / \partial x\right|^{n-1} \partial^{2} u_{z} / \partial x^{2}$. A study of this equation is currently in progress.)

Equation (5) can be rigorously applied to stress propagation in the upper mantle only when (a) displacements $u_{x}$ or $u_{y}$ are constant along a line which is much longer than relevant distances perpendicular to the line and $(b)$ displacements do not change significantly over distances comparable to the thickness of the asthenosphere. Condition $(a)$ is often satisfied for large decoupling earthquakes, where the initial motion may extend up to $1000 \mathrm{~km}$ along the plate margin. Condition (b) is less easily satisfied, as we are often interested in stress changes over distances of only a few hundred kilometers. We shall see, however, that the corrections to this approximation tend to decrease the apparent nonlinearity of the asthenosphere, so the neglect of such corrections only leads to an underestimate of $n$. Moreover, we will show in the appendix that these corrections are small even for an infinitely thick asthenosphere when $n=1$.

It is possible to avoid one or both approximations by exact calculation of the motion of the full nonlinear system. Such calculations probably cannot be done analytically, and twodimensional (or even three-dimensional) numerical models must be used. Rather than enter into the complexities of such calculations, we prefer to outline the behavior of the nonlinear system under the approximations $(a)$ and $(b)$, which are, after all, not so bad for many cases of interest. By this means we hope to arrive at a sound understanding of the behavior of stresses in a nonlinear asthenosphere. Corrections to the results presented here (which may be of great importance if an accurate determination of $n$ is desired) will generally be small.

\section{Step Function Initial Displacement}

One of the most useful solutions to (5) is the one describing the propagation of stress and displacement following a sudden displacement of magnitude $u_{0}$ at the edge of a lithospheric plate. This solution models the effect of a large decoupling earthquake, during and shortly after which the lithospheric plate may be displaced by tens of meters along a fault up to $1000 \mathrm{~km}$ long.

The boundary conditions on $u_{x}(x, t)$ for this case are

$$
u_{x}(x, 0)=0 \quad u_{x}(0, t)=u_{0}
$$

Since the boundary conditions contain no quantities with the dimensions of time, $u_{x}(x, t)$ can only be a function of the combination

$$
\eta=x /\left(\kappa t u_{0}^{n-1}\right)^{1 / 2 n}
$$

It is easily verified by direct substitution that the following solution satisfies (5) as well as both boundary conditions (7):

$$
u_{x}(x, t)=u_{0} I_{n}\left[\frac{x}{\left(\kappa t u_{0}^{n-1}\right)^{1 / 2 n}}\right]
$$

where

$$
\begin{aligned}
l_{n}(\eta)=1- & \int_{0}^{\eta / \eta_{\max }}\left(1-\zeta^{(n+1) / n}\right)^{n /(n-1)} d \zeta \\
\cdot & {\left[\int_{0}^{1}\left(1-\zeta^{(n+1) / n}\right)^{n /(n-1)} d \zeta\right]^{-1} }
\end{aligned}
$$

It is easily seen that $I_{n}(0)=1$, which satisfies (7). The dimensionless quantity $\eta$ is bounded between zero and $\eta_{\max }$, while 0 $\leq I_{n}(\eta) \leq 1 . \eta_{\max }$ is a pure number:

$$
\begin{aligned}
\eta_{\max }= & \left(\frac{n+1}{n-1}\right)^{1 / 2}(2 n)^{1 / 2 n} \\
& \cdot\left\{\int_{0}^{1}\left[1-\zeta^{(n+1) / n}\right]^{n /(n-1)} d \zeta\right\}^{-(n-1) / 2 n}
\end{aligned}
$$




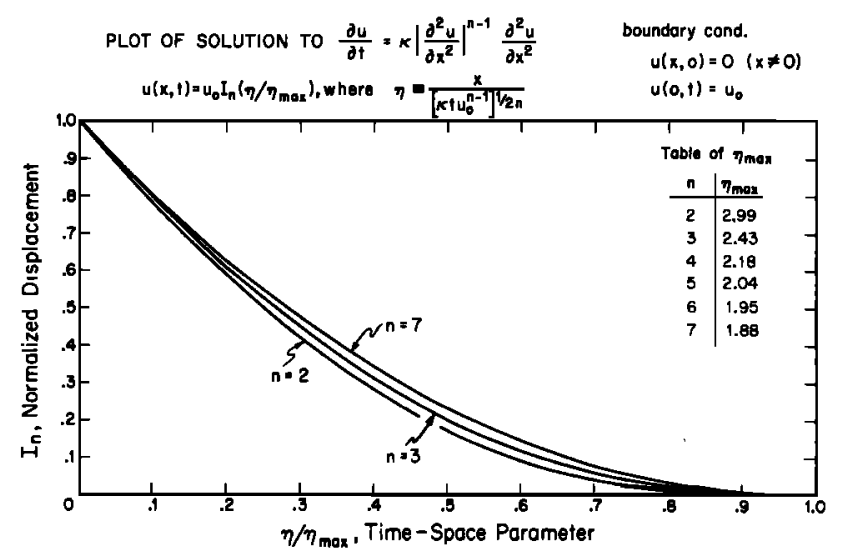

Fig. 2. The propagation of the displacement field after a sudden displacement $u_{0}$ of the boundary of a lithospheric plate overlying a nonlinear asthenosphere. See text for further details.

Except in the case $n=2$ the integrals defining $I_{n}(\eta)$ and $\eta_{\max }$ are not elementary. They are easily evaluated numerically, however, and the results are graphed in Figure 2. It is noteworthy that $I_{n}(\eta)=0$ for $\eta \geq \eta_{\max }$. Although $I_{n}(\eta)$ cannot be exactly described by an algebraic formula, Figure 2 shows that the approximation

$$
I_{n}(\eta) \simeq\left(1-\frac{\eta}{\eta_{\max }}\right)^{2}
$$

works to better than $10 \%$ for all $n$ and becomes exact in the $n \rightarrow$ $\infty$ limit. Approximation (12) is probably adequate for all geological work, although it must not be differentiated: the second derivative of $(12)$ is discontinuous at $\eta=\eta_{\max }$, whereas the exact $I_{n}(\eta)$ has continuous second derivatives everywhere.

The most important fact about $u_{x}(x, t)$ in this case is that it is a function of $\eta$ alone, falling to zero for $\eta \geq \eta_{\max }$. For small times $t \rightarrow 0, x$ must also be small to keep $\eta<\eta_{\max }$. The displacement $u_{x}$ is thus localized near $x=0$ (the edge of the plate) for small $t$. As $t$ increases, lines of constant $\eta$ (hence of constant displacement) move steadily away from the origin. As (8) shows, the velocity of expansion increases as the initial displacement increases (a feature to be expected in all nonlinear problems). The most interesting behavior is given by the $x / t^{1 / 2 n}$ factor in $\eta$. If the displacement front takes time $t_{1}$ to move distance $x_{1}$, it takes $2^{2 n} t_{1}$ to move a distance $2 x_{1}$ (e.g., for $n=3$ it takes $2^{6}=64$ times as long to move, say, $200 \mathrm{~km}$ as it does to move $100 \mathrm{~km}$ ). This is a characteristic feature of nonlinear stress propagation. Shortly after the initial displacement the front moves very rapidly away from $x=0$ but later slows down into comparative immobility.

For the sake of comparison, note that in the linear case ( $n=1)$ with boundary conditions $(7), u_{x}(x, t)$ is a function of $x /(\kappa t)^{1 / 2}$ and

$$
u_{x}(x, t)=u_{0} \operatorname{erfc}\left[\frac{1}{2} \frac{x}{(\kappa t)^{1 / 2}}\right]
$$

this $u_{x}(x, t)$ is nonzero for all $x$ when $t>0$ but is generally similar to $u_{x}(x, t)$ in the nonlinear case when considered a function of $\eta^{\prime}=x /(\kappa t)^{1 / 2}$. The major difference between the linear and nonlinear cases is the time development of the displacement front (to double $x_{1}$ in the linear case requires only $4 t_{1}$ ).

Equation (9) describes the propagation of the displacement front as a function of time and position. This equation is not directly applicable to the measurement of stress propagation following a large earthquake unless detailed geodetic information is available. Since this is not presently the case, we must fall back upon the migration pattern of aftershocks perpendicular to the fault. The locations of these aftershocks are presumably controlled by the stress in the lithosphere, $\sigma_{x}=$ $E\left(\partial u_{x} / \partial x\right)$. Equation (9) is readily differentiated, yielding

$$
\begin{array}{r}
\sigma_{x}=-\left(\frac{n-1}{n+1}\right)^{n /(n-1)}\left(\frac{1}{2 n}\right)^{1 /(n-1)} \eta_{\max }^{(n+1) /(n-1)} \\
\frac{E u_{0}}{\left[\kappa t u_{0}^{n-1}\right]^{1 / 2 n}}\left\{1-\left(\frac{\eta}{\eta_{\max }}\right)^{(n+1) / n}\right\}^{n /(n-1)}
\end{array}
$$

despite the complex appearance of this equation, it is actually quite simple, most of the coefficients being numerical constants for a given $n$. At any given time, $\sigma_{x}$ falls nearly linearly from a maximum at $x=\eta=0$ to zero at $\eta=\eta_{\max }$, or $x=\left[\kappa u_{0}^{n-1} t\right]^{1 / 2 n} \eta_{\max }$. The exact curve described by [1$\left.\left(\eta / \eta_{\max }\right)^{(n+1) / n}\right]^{n /(n-1)}$ deviates from $\left(1-\eta / \eta_{\max }\right)$ by less than $5 \%$ when $n \geq 3$.

We can reasonably expect that the stress pattern (14) begins to generate aftershocks when $\sigma_{x}$ passes through some critical range of values. Whether these aftershocks are caused directly by the changing stress in the lithosphere or are simply triggered by the arrival of the critical stress contour is irrelevant

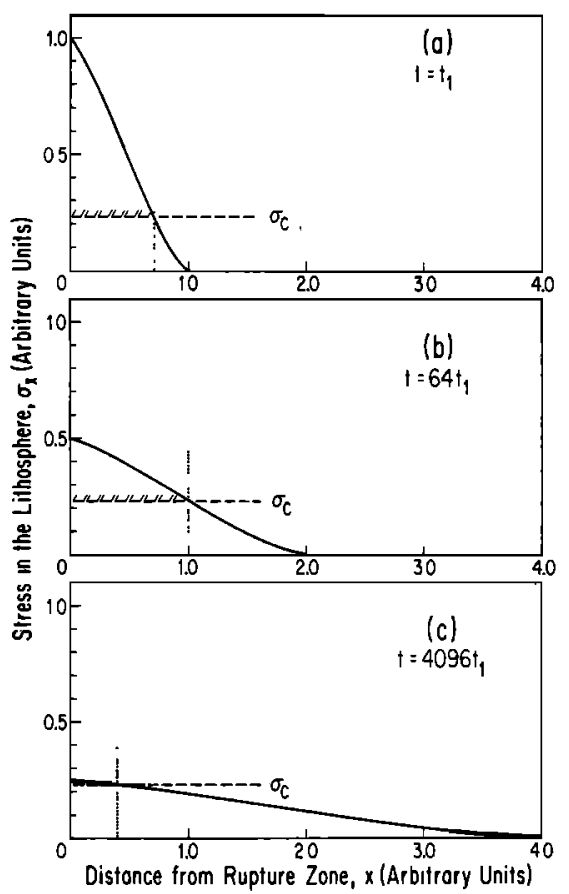

Fig. 3. Illustration of how the steady decline of stress in the lithosphere following an earthquake at the edge of a plate can lead first to an advance and then to a retreat of a stress contour. The solid curve is the stress in the lithosphere, $\sigma_{x}$, plotted versus distance $x$ from the rupture zone in accordance with (14) for $n=3$. Note, as was stated in the text, that this curve differs little from a straight line. Plot $a$ shows the stress after a time $t_{1}$ has elapsed. The stress is highest in the rupture zone, $x=0$, and it gradually dies off to zero when $x=1$. The solid curve intersects the value $\sigma_{c}$ at a distance shown by the vertical dotted line. The stress exceeds $\sigma_{c}$ everywhere that the horizontal $\sigma_{c}$ line is decorated by diagonal slashes. Plot $b$ shows the situation some time later. We see that the stress declines to $\sigma_{c}$ somewhat further away from the rupture zone, at $x=1$. Plot $c$ is drawn at a time just before the stresses everywhere drop below $\sigma_{c}$. It is apparent that the stress declines to $\sigma_{c}$ much closer to the rupture zone than before. In the last instant before the stress drops below $\sigma_{c}$, the $\sigma_{c}$ contour will reach $x=$ 0 , as described in the text. 
TABLE 1. Coefficients of Equations Describing Constant $\sigma_{x}$ Contours for Various Values of $n$

\begin{tabular}{cccccc}
\hline$n$ & $\eta / \eta_{\text {max }}$ & $t_{m} / t_{0}$ & $c_{\text {kappa }}$ & $c_{\text {aIgma }}$ & $c_{\text {distance }}$ \\
\hline 1 & $\ldots$ & 0.368 & 1.36 & 0.484 & 1.649 \\
2 & 0.397 & 0.100 & 5.04 & 0.496 & 4.480 \\
3 & 0.439 & $2.60 \times 10^{-2}$ & $2.62 \times 10^{1}$ & 0.495 & 4.188 \\
4 & 0.456 & $6.65 \times 10^{-8}$ & $1.57 \times 10^{2}$ & 0.493 & 4.101 \\
5 & 0.466 & $1.69 \times 10^{-3}$ & $9.84 \times 10^{2}$ & 0.496 & 4.064 \\
6 & 0.472 & $4.26 \times 10^{-4}$ & $6.33 \times 10^{s}$ & 0.499 & 4.044 \\
7 & 0.476 & $1.07 \times 10^{-4}$ & $4.35 \times 10^{4}$ & 0.498 & 4.032 \\
\hline
\end{tabular}

See text for full explanations.

$\kappa=c_{\mathrm{kappa}}\left(x_{m}{ }^{2 n}\right) /\left(u_{0}{ }^{n-1} t_{0}\right)$ (see equation (20)).

$\sigma_{x} / E=c_{\text {sigma }}\left(u_{0} / x_{m}\right)$ (see equation (21))

$x / x_{m}=c_{\text {dlatance }}\left[\left(t / t_{0}\right) \ln ^{\prime}\left(t_{0} / t\right)\right]^{1 / 2}$ for $n=1$ (see equation (19)).

$x / x_{m}=c_{\text {dlatance }}\left(t / t_{0}\right)^{1 / 2 n}\left[1-\left(t / t_{0}\right)^{(n-1) / 2 n^{2}}\right]^{n /(n+1)}$ for $n>1$ (see equation (18))

for our argument. All that we require is that aftershocks occur frequently only when the stress exceeds some critical value. We shall approximate this transition by a step function, assuming that aftershocks occur only when $\sigma_{x}$ exceeds a threshold value $\sigma_{x}^{c}$. The pattern of aftershock activity should thus be controlled by the position of the $\sigma_{x}(x, t)=\sigma_{x}^{c}$ contour. Between the contour line and $x=0, \sigma_{x}>\sigma_{x}{ }^{c}$ and aftershocks should occur. Outside of the line $\left(\sigma_{x}<\sigma_{x}^{c}\right)$, little activity should be apparent. An equation for lines of constant $\sigma$ is derived by setting

$$
d \sigma=\frac{\partial \sigma}{\partial x} d x+\frac{\partial \sigma}{\partial t} d t=0
$$

thus

$$
\frac{d x}{d t}=-\frac{\partial \sigma / \partial t}{\partial \sigma / \partial x}
$$

Using (14) to evaluate the partial derivatives in (15), a differential equation is obtained for the lines of constant $\sigma_{x}$. This differential equation can be integrated for each $n$ and an algebraic equation for the curve obtained. A general feature of these curves is that each begins from $x=t=0$ and moves toward larger $x$ as $t$ increases until it reaches a maximum distance $x_{m}$ from the origin at time $t_{m}$. The curve then retreats from $x_{m}$, eventually returning to $x=0$ at time $t_{0}$ as illustrated in Figure 3. This retreat, at first sight rather odd, is due to the general decrease of $\sigma_{x}$ with time. In the initial stages of the expansion, $\sigma_{x}$ is everywhere large, so as the front $\eta=\eta_{\max }$ expands, the $\sigma_{x}{ }^{c}$ contour expands also. After a while, however, $\sigma_{x}$ declines to the point where $\sigma_{x}(0, t)$ is very little larger than $\sigma_{x}{ }^{c}$. At this stage, retreat begins, until at time $t_{0}$ the maximum stress is $\sigma_{x}{ }^{c}$ and must therefore occur at $x=0$. For times $t>t_{0}$, $\sigma_{x}<\sigma_{x}{ }^{c}$ everywhere, and no aftershocks will occur.

The maximum extension of any $\sigma_{x}(x, t)=$ const contour occurs when

$$
\frac{\eta}{\eta_{\max }}=\left(\frac{n-1}{2 n}\right)^{n /(n+1)}
$$

The value of $\eta / \eta_{\max }$ is nearly 0.5 for all $n$ (see Table 1). The $\sigma(x, t)=$ const contours can be parameterized by the maximum excursion $x_{m}$ and the time $t_{0}$ required for the curve to retreat to the origin. The time $t_{0}$ can be directly read off from (14) by setting $x=\eta=0$ and $\sigma_{x}=$ const. The ratio between $t_{m}$ and $t_{0}$ is derived by equating $\sigma\left(0, t_{0}\right)$ and $\sigma\left(x_{m}, t_{m}\right)$ and using (14) and (16).

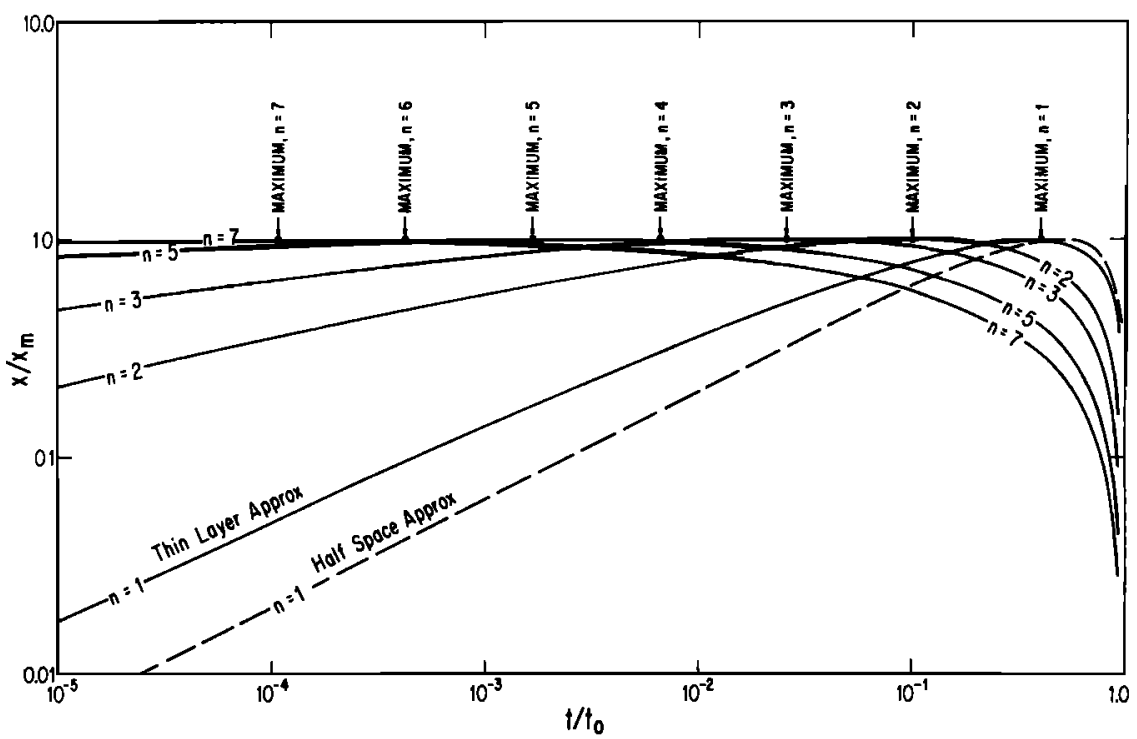

Fig. 4. The position of a given stress contour in the lithospheric plate as a function of time after an initial stepwise displacement of the plate boundary. The distance $x$ is normalized by the maximum excursion $x_{m}$, and the time $t$ is normalized by the time $t_{0}$ for the stress contour to return to the plate boundary. Contours are drawn for stress-strain rate power laws with $n=1$ to 7 . The time at which each contour reaches its maximum excursion is indicated. The solid curves are computed for the thin asthenosphere approximation. The dashed curve is for an infinitely thick asthenosphere (a half space) when $n=1$. Note that it differs only slightly from the solid $n=1$ curve. 


$$
\frac{t_{m}}{t_{0}}=\left(\frac{n+1}{2 n}\right)^{2 n^{2 /(n-1)}}
$$

$\left(t_{m} / t_{0}=1 / e\right.$ in the linear, $n=1$ case). Values of this ratio for various $n$ can be found in Table 1. The general effect of larger $n$ is to lengthen the interval of time between $t_{m}$ and $t_{0}$ relative to $t_{m}$. Thus for $n=3$ it takes 38 times as long to retreat from $x_{m}$ to $x=0$ as it originally took to move out to $x_{m}$ (compare this to 2.7 times longer for retreat than advance when $n=1$ ). In applications to aftershock migration patterns it is this slow retreat which is observed, since the initial expansion to $x_{m}$ occurs rapidly. We shall see that observed aftershock patterns retreat far more slowly than could be expected for a linear asthenosphere.

Supposing that $x_{m}$ and $t_{0}$ are known (as they would be from a study of aftershock migration), the form of $\sigma_{x}(x, t)=$ const curves is

$$
\begin{aligned}
\frac{x}{x_{m}}=\left(\frac{2 n}{n-1}\right)^{n /(n+1)}\left(\frac{2 n}{n+1}\right)^{n /(n-1)}\left(\frac{t}{t_{0}}\right)^{1 / 2 n} \\
\cdot\left[1-\left(\frac{t}{t_{0}}\right)^{(n-1) / 2 n^{2}}\right]^{n /(n+1)}
\end{aligned}
$$

This curve is plotted in log-log coordinates in Figure 4. The log-log presentation has the advantage that a change in $x_{m}$ or $t_{0}$ appears only as a change in origin, the shape of the curve being invariant. This sort of plot is also the most useful for comparison with observational data. Note that for a linear asthenosphere

$$
\frac{x}{x_{m}}=(e)^{1 / 2}\left[\left(\frac{t}{t_{0}}\right) \ln \left(\frac{t_{0}}{t}\right)\right]^{1 / 2}
$$

This curve is also plotted in Figure 4. The values of $x_{m}$ and $t_{0}$ can be used to compute other parameters of interest (once $n$ is known from fitting $x / x_{m}$ versus $t / t_{0}$ curves):

$$
\begin{aligned}
\kappa & =\left(\frac{2 n}{n-1}\right)^{2 n^{2} /(n+1)}\left(\frac{2 n}{n+1}\right)^{2 n^{2} /(n-1)}\left(\frac{1}{\eta_{\max }}\right)^{2 n} \frac{x_{m}^{2 n}}{u_{0}^{n-1} t_{0}} \\
& =\frac{e}{2} \frac{x_{m}^{2}}{t_{0}} \quad n=1
\end{aligned}
$$

and

$$
\begin{aligned}
\frac{\sigma_{x}^{c}}{E} & =\left[\frac{\eta_{\max }^{2 n}}{2 n}\left(\frac{n-1}{2 n}\right)^{2 n^{2 /(n+1)}}\right]^{1 /(n-1)}\left(\frac{u_{0}}{x_{m}}\right) \\
& =\left(\frac{2}{e \pi}\right)^{1 / 2}\left(\frac{u_{0}}{x_{m}}\right) \quad n=1
\end{aligned}
$$

The values of the coefficients in these equations are given in Table 1 for various $n$. The most notable feature is that $\sigma_{x}{ }^{c} / E \simeq$ $0.5\left(u_{0} / x_{m}\right)$, regardless of $n$. This result is easily understood on geometrical grounds. As a result, the maximum extension of the aftershock pattern away from the fault gives no information about the rheology of the asthenosphere, being mainly controlled by the value of $\sigma_{x}^{c}$ and the initial displacement $u_{0}$. The initial displacement $u_{0}$ cannot be calculated if only $x_{m}$ and $t_{0}$ are known. Both $k$ and $\sigma_{x}{ }^{c}$ are functions of $u_{0}$, so in order to estimate $\kappa$ and $\sigma_{x}^{c}$ we must make a reasonable guess about $u_{0}$ (usually several meters). Again, geodetic information would determine $u_{0}$ unambiguously.

\section{Steady Displacement of the Plate Boundary}

The preceding section has shown that if the asthenosphere is non-Newtonian, $n>1$, the stress and displacement fields due to an earthquake at the plate edge are strongly restricted to that edge. This effect is apparent even for $n=1$ (as noted by Bott and Dean [1973]). The velocity at which a displacement contour recedes from the plate edge decreases as $1 / t^{1-(1 / 2 n)}$, falling away like $1 / t$ for large $n$ (as opposed to $1 /(t)^{1 / 2}$ for $n=$ 1). The typical behavior is for a displacement contour to recede rapidly from the plate edge, then quickly slow down beyond some characteristic distance (which may be of the order of a few hundred kilometers from the plate edge). If the displacement contour takes $1 \mathrm{yr}$ to recede $100 \mathrm{~km}$ from the plate edge, then it will take $10^{2 n}$ yr to recede $1000 \mathrm{~km}$. When $n$ $=3$, this time will be $10^{6} \mathrm{yr}$.

This behavior leads to some puzzlement about how the motion in the interior of a plate can be affected by forces applied to its edges. Estimates by Elsasser [1967] show that there is no problem for $n=1$, but if $n=3$ (as suggested by the analysis later on in this paper), then the results of the previous section show that an earthquake at the plate edge cannot affect its interior unless the plate is only a few hundred kilometers in size.

The purpose of this section is to show that the net effect of a series of earthquakes, whose displacements one following the other can be considered to produce an average velocity of the plate edge, is to propagate the average motion rather rapidly into the plate's interior. This effect is due to the nonlinear superposition of stresses inherent in (1). Thus suppose that the displacement caused by one earthquake propagates outward as $t^{1 / 2 n}$, leaving a stress $\sigma_{i}{ }^{(1)}$ in the upper mantle. A second earthquake occurring later adds to this a stress $\sigma_{i j}^{(2)}$. The strain rate induced by this superposition is not simply the sum of the strain rates due to each stress field alone but is of order $\dot{\boldsymbol{\epsilon}} \sim$ $\left(\sigma^{(1)}+\sigma^{(2)}\right)^{n}$. For $n>1$ this strain rate is larger than that given by simple addition. Thus each succeeding earthquake's strain field is boosted along by that of the preceding ones, resulting in a net pattern which moves along quickly in comparison to that of a single earthquake.

The detailed analysis of the stress and strain fields due to a sequence of discrete earthquakes at the plate edge is a formidable mathematical problem when $n \neq 1$. Rather than attempt such a problem, we shall go to the extreme of assuming that earthquake events are so frequent that on the average the motion of the plate boundary can be represented as a steady displacement, $u_{x}(0, t)=k t$, where $k$ is the average velocity of the plate boundary. The stress and displacement fields derived from this boundary condition will apply to the actual lithospheric plate only at some distance from its edge. Nearer to the edge than this distance the stress and displacement will be fluctuating, dominated by contributions from individual earthquakes. These earthquakes produce small, sudden displacements of the lithospheric plate. Such displacements involve stresses far higher than the average stresses in the lithosphere, and these stresses override the influence of the average stresses in (1). Thus for a short time following an earthquake, the stress and displacement fields expand as described in the previous section, widening in area as well as weakening as time goes on. Eventually, the stresses derived from the sudden displacement become comparable to the average stresses in the lithosphere (this sequence has been studied in detail by Bott and Dean [1973] for $n=1$ ). At this point the nonlinearity of (1) mixes the effect of the stress fields in a complex way. The distance from the edge of the plate to this point can be estimated roughly by equating it to the maximum excursion of the shear stress contour whose magnitude equals that of the average shear stress, $\left\langle\sigma_{x z}\right\rangle$. This excur- 
sion can be found by methods identical to those which were used to find the maximum excursion $x_{m}$ of a $\sigma_{x}$ contour in the last section. The result is

$$
x_{11 \mathrm{~m} / \mathrm{t}} \simeq\left(\frac{h_{1} E u_{0}}{2\left\langle\sigma_{x z}\right\rangle}\right)^{1 / 2}
$$

or since the average displacement per event, $u_{0}$, is equal to the average velocity $k$ multiplied by the time $\tau$ between events,

$$
x_{11 \mathrm{mit}} \simeq\left(\frac{h_{1} E k \tau}{2\left(\sigma_{x z}\right\rangle}\right)^{1 / 2}
$$

The average shear stress $\left\langle\sigma_{x z}\right\rangle$ can be written in terms of $k$ by means of (4). Thus

$$
x_{1 \mathrm{ImIt}} \simeq\left(\frac{\kappa^{1 / n} k^{(n-1) / n} \tau}{2}\right)^{1 / 2}
$$

Furthermore, we can use (20) to determine $\kappa$ from the observables $x_{m}$ and $t_{0}$. Note that $x_{m}$ and $t_{0}$ are the parameters of any stress contour that we happen to observe: they need not refer to any specific stress contour, since the ratio $x_{m}{ }^{2 n} / t_{0}$ is the same for all stress contours. Thus we arrive at the most practical form of (22),

$$
x_{1 \mathrm{ImIt}} \simeq\left(c_{\mathrm{kappa}} \frac{\tau}{t_{0}}\right)^{1 / 2 n} \frac{x_{m}}{2^{1 / 2}}
$$

where $c_{\text {kappa }}$ is defined in Table 1 . In nature, $x_{11 \mathrm{~m} \text { it }}$ generally turns out to be less than $500 \mathrm{~km}$.

As might be expected, when $\tau$ is large, earthquakes are infrequent but involve large displacements. Hence their influence extends a greater distance into the plate than when earthquakes are frequent but involve small displacements.

In either case, the effects of individual earthquakes merge into the average stress field for $x>x_{11 m 1 t}$, and in this region the steady displacement approximation is valid. This approximation is thus correct for the interiors of lithospheric plates or for averages over long intervals of time.

The boundary conditions for a plate whose edge begins moving at time $t=0$ with velocity $k$ are

$$
\begin{aligned}
& u_{x}(x, 0)=0 \\
& u_{x}(0, t)=k t
\end{aligned}
$$

Steady motion, begun long ago, is a special case of this solution (the $t \rightarrow \infty$ limit). It is readily verified by substitution into (5) that $u_{x}(x, t)$ is of the form

$$
u_{x}(x, t)=k \operatorname{tg}(\phi)
$$

where

$$
\phi=\frac{1}{\left(k^{n-1} \kappa\right)^{1 / 2 n}} \frac{x}{t^{1 / 2}}
$$

The function $g(\phi)$ is a solution to the ordinary differential equation

$$
\left(\frac{d^{2} g}{d \phi^{2}}\right)^{n}=g(\phi)-\frac{\phi}{2} \frac{d g}{d \phi}
$$

The boundary conditions (26) require $g(\phi)=1$. Physical solutions to (29) fall to zero for some finite $\phi_{\max }, g\left(\phi_{\max }\right)=0$. Moreover, $g(\phi)=0$ for $\phi>\phi_{\max }$. Equation (29) was integrated numerically, and the results are graphed in Figure 5. The function $g(\phi)$ looks very similar to $I_{n}(\eta)$ and in fact can be well approximated by

$$
g(\phi) \simeq\left(1-\frac{\phi}{\phi_{\max }}\right)^{2}
$$

which is correct to within $10 \%$ for $n \geq 2$. Equation (30) becomes exact in the limit $n \rightarrow \infty$, when $\phi_{\max }=2^{1 / 2}$. The values of $\phi_{\max }$ given in Figure 5 may be as much as $20 \%$ too low due to the numerical difficulty in following out the long tail of $g(\phi)$ as it approaches zero. The curve of $g(\phi)$ versus $\phi / \phi_{\max }$ is accurate to better than $1 \%$.

Equation (27) is identical in form to the $n=1$ solution [Crank. 1956]

$$
u_{x}(x, t)=k t\left\{\left(1+\frac{x^{2}}{2 \kappa t}\right) \operatorname{erfc}\left(\frac{x}{2(\kappa t)^{1 / 2}}\right)-\frac{x}{(\pi \kappa t)^{1 / 2}} e^{-x^{2 / 4 k t}}\right\}
$$

The complicated expression enclosed in brackets is a function only of $x / t^{1 / 2}$, just as $g$ is in (27). Thus aside from some details in the shape of the displacement front, the nonlinear solutions behave very much like the linear solution.

We found in the previous section that a single sudden displacement at the edge of a plate generates a stress and displacement front which rapidly slows down as it moves into the plate. This effect becomes more marked as the nonlinearity increases. Thus an individual earthquake's influence dies out rather rapidly away from its epicentral region with a nonlinear asthenosphere. On the other hand, (27) shows that a steady motion of the plate boundary is rapidly propagated across the lithospheric plate. As $n$ increases, the motion. of the plate becomes less subject to perturbations from individual earthquake events and more accurately reflects the average, longterm forces acting upon it.

\section{Part II: Determination of the Rheology of the ASTHENOSPHERE}

\section{Generalities}

We have seen that the pattern of stress in the lithosphere after a large decoupling earthquake is strongly influenced by the rheology of the underlying asthenosphere. If we could observe the stress (or displacement) pattern, then we could evidently measure the rheology. Lacking any direct way of determining lithospheric stresses, we are forced into the somewhat tricky business of using the occurrence of aftershocks to delineate the motion of the stress contours. We assume that aftershocks occur only when the regional stresses exceed some critical value $\sigma_{x}{ }^{c}$ (this assumption is not crucial; if aftershocks begin to occur over some range of stresses, rather than at a sharp threshold, the only effect is to smear out the aftershock

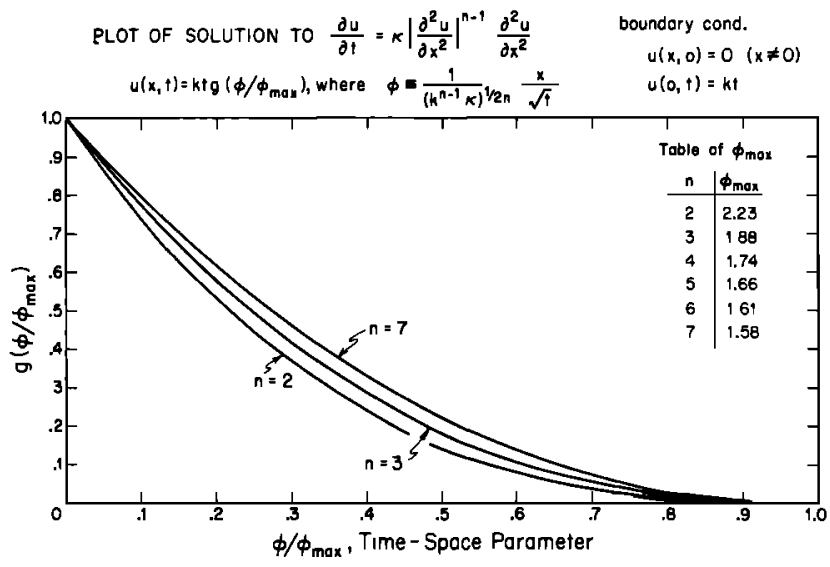

Fig. 5. The propagation of the displacement field during a steady displacement of the boundary of a lithospheric plate overlying a nonlinear asthenosphere. See text for further details. 


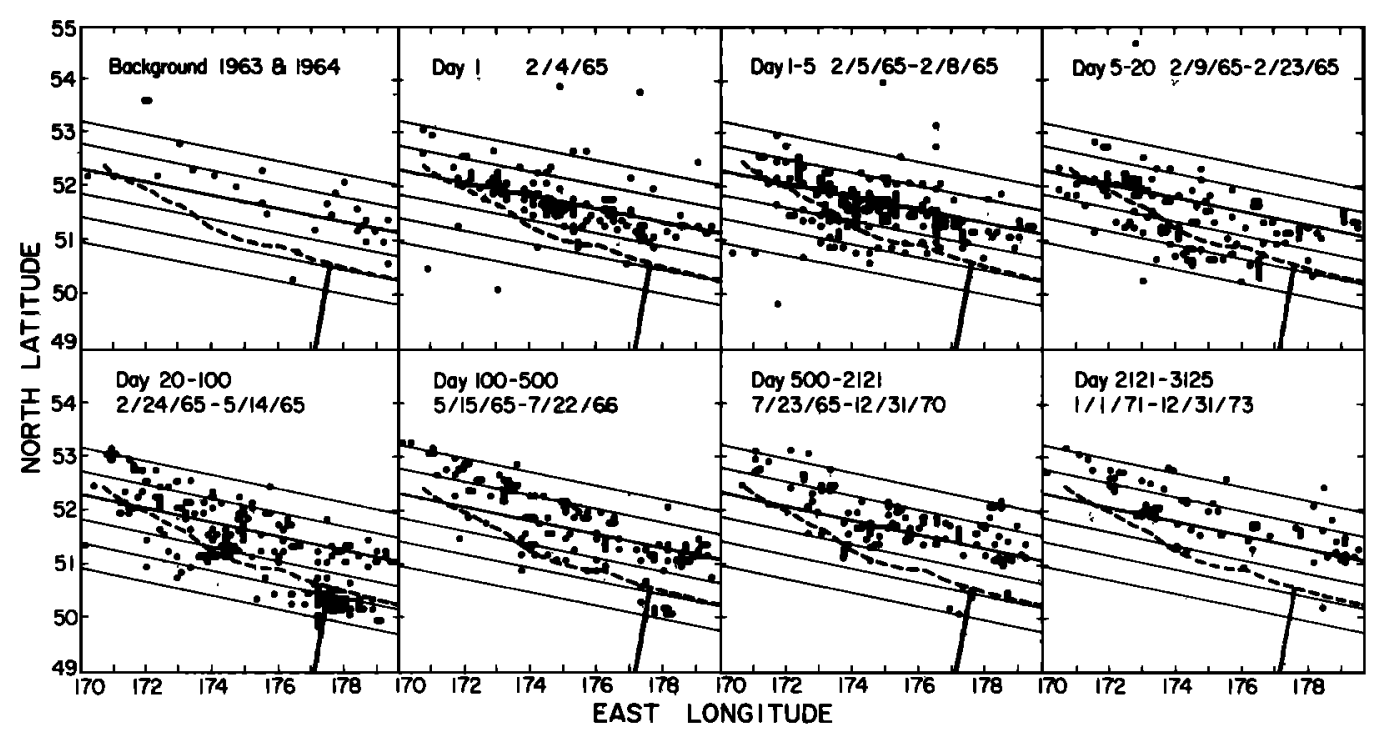

Fig. 6. Plot of epicentral positions of aftershocks with $M \geq 4.5$ following the February 4, 1965, Rat Island earthquake. The heavy oblique straight line in the day 1 frame is a least squares fit to the epicenters of the aftershocks occurring during day 1. The lighter parallel lines are at intervals of $50 \mathrm{~km}$ away from the least squares line. This grid is reproduced in all later frames so that epicenters of aftershocks occurring at later times can be compared to the frame of reference established on the first day. The heavy dashed line represents the position of the Aleutian trench, while the dotted line follows the trend of the Rat fracture zone. Note the concentration of aftershocks at the intersection of these features between day 20 and day 100 .

pattern slightly). We then proceed, as in the section on step function initial displacement in part $I$, to convert the apparent motion of the aftershock pattern into rheological information.

In order to obtain interpretable data, we seek an earthquake which satisfies our various approximations as completely as possible. As in any laboratory experiment we must isolate the phenomenon that we wish to study, eliminating all extraneous complications. Once the rheology of the asthenosphere is established, we can go on to analyze more complex situations where many parameters enter into the final result. For the present, however, we shall be content with eliminating as many such parrameters as possible.

These considerations make the selection of an appropriate earthquake an important part of the measurement. To guide the selection, we set down the following list of criteria for a usable event.

1. It must be a large decoupling earthquake with many aftershocks (in order to get enough statistical data on the aftershock pattern).

2. The initial displacement must take place along a line exceeding $500 \mathrm{~km}$ in length (in order to satisfy the one-dimensional approximation of equation (4)).

3. The earthquake should be isolated, with no other major earthquakes occurring near it for several decades before or after the event studied (this allows time for shear stresses in the asthenosphere to reach low levels before the earthquake).

4. The principal displacement of the edge of the lithospheric plate should be horizontal, either strike-slip or lowangle thrust (otherwise, equation (4) does not apply).

5. The aftershocks should occur in the oceanic plate, since the stress propagation theory probably does not apply to the overthrusting plate without some modification.

If we add to these criteria the practical consideration that aftershocks were adequately recorded and located only after 1962, we see that we have few events to choose from. Preliminary analyses were carried out on the $1963 \mathrm{~K}$ urile Islands event, the 1964 Alaska earthquake, and the 1965 Rat Island earthquake. Of these, the $\mathrm{K}$ urile Islands earthquake was rejected on count 3 (other major events occurred in that region during 1958, 1968, and 1969), while the Alaska earthquake was rejected on count 5 (nearly all of the aftershocks were very shallow and occurred inland of the Aleutian trench). In spite of these objections, both earthquakes showed aftershock migration patterns consistent with the results of the Rat Island analysis, although they were not as clear-cut as those of $R$ at Island.

\section{Analysis of the 1965 Rat Island Earthquake}

The 1965 Rat Island Earthquake occurred at 05h 01m UT on February 4, 1965. Although assigned magnitude $7 \frac{3}{4}$, it was apparently larger than the 1963 Kurile Islands earthquake ( $M$ $=8.2$ ) in terms of seismic moment [ $W u$ and Kanamori, 1973]. During the 45-day period following the main event the U.S.C.G.S. (U.S. Coast and Geodetic Survey) located 870 aftershocks [Jordan et al., 1965], most of which occurred in a rectangular zone $650 \mathrm{~km}$ long and $200 \mathrm{~km}$ wide. The last earthquake of comparable magnitude to occur in this area was on July 14, 1940 ( $M=73$; Gutenberg and Richter [1954]), $25 \mathrm{yr}$ before the 1965 event. No other earthquakes of comparable size have occurred in this region since 1965. The fault plane solutions for the main event [Wu and Kanamori, 1973] show that the motion was nearly horizontal, dipping only $18^{\circ}$. The motion was an almost equal mixture of strike-slip and thrust. The initial rupture surface was $500 \mathrm{~km}$ long, striking $\mathrm{N} 70^{\circ} \mathrm{W}$. After the first day, many aftershocks were located at depths up to $50 \mathrm{~km}$ in the oceanic plate south of the Aleutian trench. The 1965 Rat Island event thus satisfies all the criteria laid down in the previous section.

Only aftershocks of $M \geq 4.5$ were used in this analysis, in order to eliminate possible bias due to seismograph site chaices. All locations were obtained from the U.S.C.G.S. preliminary listings, which are sufficiently accurate for our pur- 


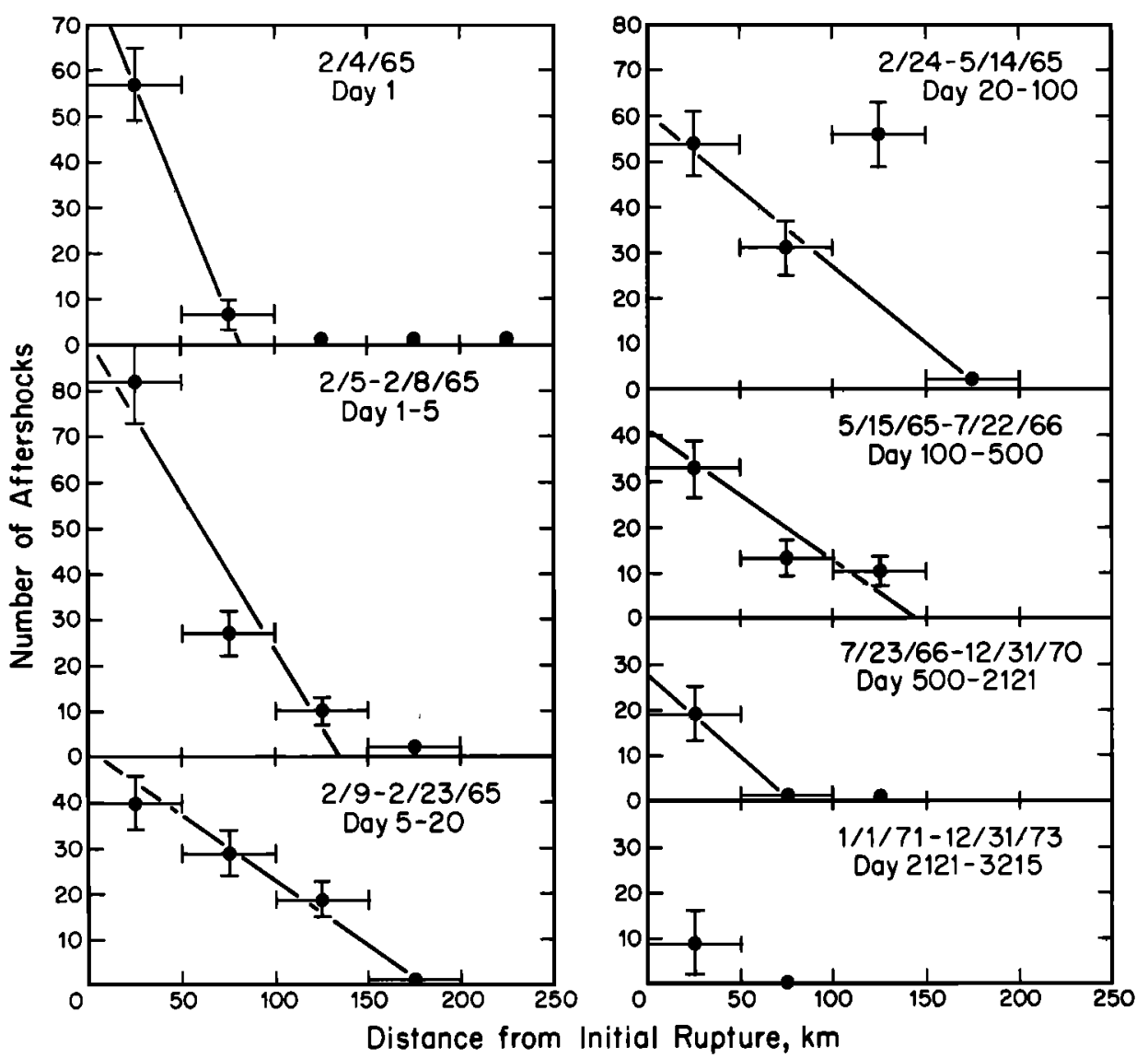

Fig. 7. Aftershock migration patterns of the February 4, 1965, Rat Island earthquake. The number of aftershocks plotted is the number observed in the stated interval minus a background computed from the seismic activity in 1963 and 1964. Errors on the number of aftershocks are statistical, while those on the distance reflect the 50-km bins chosen. This figure only includes aftershocks which occurred south of the zone defined by the aftershocks of the first day.

poses. (The rms location error is about $25 \mathrm{~km}$, from a comparison of U.S.C.G.S. and International Seismological Centre (ISC) positions. This is only half the bin width used in our analysis and so can be neglected.) The position of the initial rupture (along which we assume that the initial displacement $u_{0}$ of the plate 'edge' occurs) was defined by a least squares line fit to the aftershocks of the first day (February 4, 1965). The FWHM (full width at half maximum) of the aftershock distribution on this day was about $50 \mathrm{~km}$, and the equation of the line was latitude $=-0.127$ (longitude) $+73.948^{\circ}$, where the longitude is reckoned east of Greenwich. Aftershocks extended for about $650 \mathrm{~km}$ along this line, which was $50-75 \mathrm{~km}$ north of the Aleutian trench and nearly parallel to it. All aftershocks of $M \geq 4.5$ lying between $49^{\circ} \mathrm{N}$ and $55^{\circ} \mathrm{N}$ latitude and $170^{\circ} \mathrm{E}$ and $180^{\circ} \mathrm{E}$ longitude were used in this study.

The distance of each aftershock from the least squares line (thus representing the distance from the line of initial rupture) was computed, and the results binned in $50-\mathrm{km}$ intervals. The aftershock pattern retained its initial rectangular form as it spread south into the oceanic plate, thus making this binning procedure meaningful. The aftershock pattern resulting from this procedure is shown in Figure 6, where the 5-yr period $1965-1970$ is divided into six logarithmically increasing time intervals (this choice ensures that roughly equal numbers of aftershocks will occur in each interval). The last graph in Figure 6 contains aftershock data for 1970-1973, the latest data available. The errors assigned to each point are statistical errors only, derived on the basis of the number of earthquakes seen minus the number estimated to form part of the back ground activity (calculated from 2 yr of activity in 1963-1964). Note that the position of the Aleutian trench has no apparent effect on the aftershock pattern, indicating that the aftershocks do not occur on the fault plane. This is confirmed by a study of the depths of the aftershocks.

The expected pattern of aftershock migration into the plate, a halt, then a retreat back to the plate margin, is readily seen in Figure 6. Since the edge of the Pacific plate was pulled outward in the main earthquake, we expect the stress field induced in the plate to be extensional. Focal mechanisms determined for aftershocks in the Pacific plate did, in fact, indicate normal faulting [Stauder, 1968]. The number of aftershocks as a function of distance from the initial rupture falls linearly to zero at some distance from the rupture (but note that the average magnitude of aftershocks in a given bin shows no decided trend as a function of distance). This suggests that the number of aftershocks (but not their average magnitude) is roughly proportional to the value of the regional stress. The only major exception to this rule occurred between February 24, 1965, and May 14, 1965, at a distance of $100 \mathrm{~km}$ south of the rupture zone. Most of the anomalous aftershocks occurred in a small region about $75 \mathrm{~km}$ across near the intersection of the Rat fracture zone and the Aleutian trench, suggesting that the large number of events may be due to local causes. The position of the edge of the aftershock pattern is independent of whether we keep these extra events or subtract them, so we will not go further into this problem. The position of the critical stress 
contour is determined by linearly extrapolating the number of aftershocks to zero, as shown in Figure 7. The reader may readily verify that other methods of defining the edge of the aftershock pattern yield the same results within the assigned errors. The position of this contour as a function of time is plotted in Figure 8. Errors of $\pm 25 \mathrm{~km}$ were assigned after a study of the detailed aftershock patterns. The curves fit to the data in Figure 8 (which are replotted from Figure 4) make it apparent that a linear asthenosphere cannot describe the data. Further than the bare fact of nonlinearity, however, we cannot yet go. All the curves for $n \geq 2$ are consistent with the data within the rather large error bars. If $n$ were much greater than 6 , however, the retreat of the aftershock pattern would be far slower than that observed here. We thus assign $n$ the value $4 \pm$ 2 as representing the most likely $n$ consistent with this analysis. Summarizing our results for the 1965 Rat Island aftershock migration patterns, we find

$$
\begin{gathered}
n=4 \pm 2 \quad x_{m}=175 \pm 25 \mathrm{~km} \\
t_{0}=2600 \pm 500 \text { days }
\end{gathered}
$$

Note that $x_{m}$ is not much larger than typical values for the thickness of the asthenosphere, $50-100 \mathrm{~km}$. This, in turn, means that assumption $(b)$ in the section on fundamental equations in part I may not be strictly valid for the Rat Island earthquake. The appendix shows, however, that the stress propagation pattern is insensitive to asthenosphere thickness for $n=1$. Furthermore, increasing the thickness of the asthenosphere increases the disagreement between the $n=1$ prediction and the data, as the dashed line in Figure 8 shows. The case for $n \neq 1$ is thus strengthened. Moreover, the insensitivity of the results to the asthenosphere's thickness for $n=1$ makes it plausible that our curves are not very sensitive to the asthenosphere's thickness even when $n>1$. This presumption must be backed up by detailed numerical computation, of course, especially if we are interested in a precise value for $n$. The poor quality of the data at the present time does not justify the labor involved in such calculations, especially since our prime conclusion that $n \neq 1$ is already well established.

\section{Inferences About Plate Motion}

The foregoing analysis has provided strong evidence that the rheology of the asthenosphere is nonlinear. The observed parameters $x_{m}$ and $t_{0}$ can be used as described in the section on initial displacement in part I to calculate quantities which are of more direct geological interest than $x_{m}$ and $t_{0}$ alone. Thus
(20) can be used to compute $\kappa$, provided $u_{0}$ is known. The source mechanism of the February 4 main event indicated an average displacement of $2.5 \mathrm{~m}$ [ $W u$ and Kanamori, 1973] over the $500-\mathrm{km}$-long rupture. This displacement could have been increased somewhat by postseismic creep. However, the average subduction velocity of $6 \mathrm{~cm} / \mathrm{yr}$ [Le Pichon, 1968] combined with the $25-y r$ interval between comparable events at this location requires an average $u_{0}$ of $1.5 \mathrm{~m}$, indicating that the $2.5-\mathrm{m}$ figure is probably of the correct order of magnitude.

Choosing Young's modulus $E=1.6 \times 10^{6}$ bar for the lithosphere [Bullen, 1965], we have computed $\kappa$ for $n=2,4$, and 6 . The results appear in Table 2. In order to compare these results to laboratory measurements on the creep of olivine, we have used (6) in conjunction with the low-stress creep measurements of Kohlstedt and Goetze [1974] to compute a theoretical value for $\kappa$. For this calculation we have assumed a lithospheric thickness $h_{1}=70 \mathrm{~km}$ and asthenospheric thickness $h_{2}=50 \mathrm{~km}$, and we calculate $A$ for the various $n$ by setting $\dot{\epsilon}=10^{-\theta} \mathrm{s}^{-1}$ for $\sigma=100$ bar at a standard temperature of $1300^{\circ} \mathrm{C}$ (calculated from the data of Kohlstedt and Goetze [1974]). The remarkable agreement between the calculated and observed values of $\kappa$ is apparent from Table 2. The discrepancies of 1 or 2 orders of magnitude in the results can readily be accounted for if temperatures in the asthenosphere differ from the assumed $1300^{\circ} \mathrm{C}$ by only a few hundred degrees, since a temperature increase of $100^{\circ} \mathrm{C}$ raises $\kappa$ by an order of magnitude. There is a tendency for the calculated and observed $\kappa$ to agree better for the smaller $n$ values, indicating that the true value of $n$ probably lies near the low end of the 2-6 range.

The fact that the observed stress propagation coefficient comes very close to values calculated from laboratory creep data is in itself strong evidence that we have correctly interpreted the aftershock migration patterns.

Another of the parameters which we can compute from our model of aftershock generation and stress propagation is the critical regional stress required to generate frequent aftershocks (equation (21)). The result, $11 \pm 2$ bar, is in line with current thinking [Molnar and Wyss, 1972] on the stress drop which occurs during lithospheric earthquakes.

The effective viscosity of the asthenosphere at a shear stress level (about 2 bar) corresponding to the $6-\mathrm{cm} / \mathrm{yr}$ plate velocity in this region is easily derived from the formula $\eta_{\text {efr }}=$ $\left(h_{1} h_{2} E / k\right)(k / \kappa)^{1 / n}$. Its value is nearly $4 \times 10^{19} \mathrm{P}$ for all values of $n$.

Last, the distance to which the influence of individual earth-

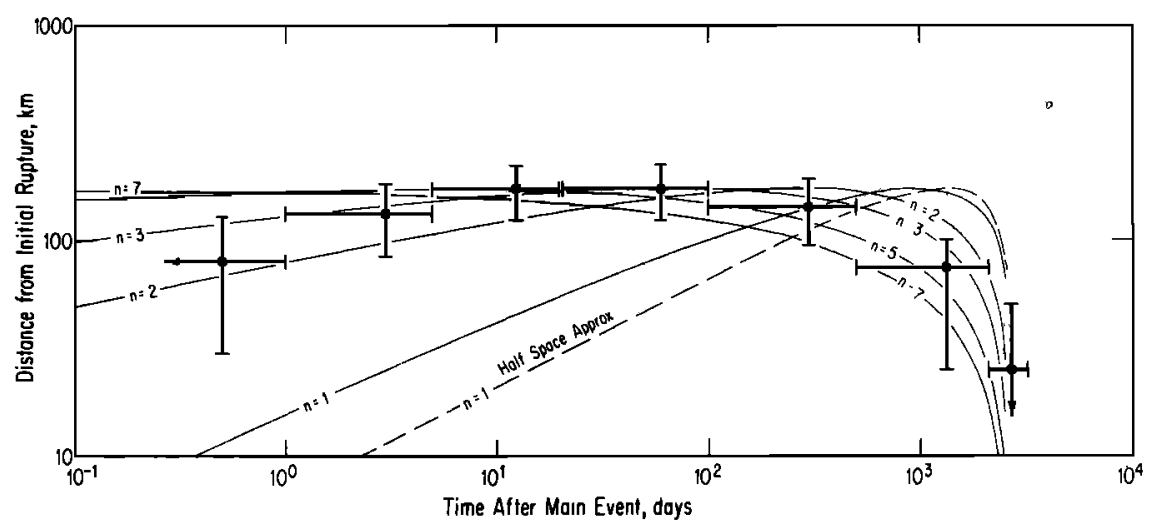

Fig. 8. Position of the aftershock front as a function of time after the main event on February 4, 1965. Note particularly that neither $n=1$ curve can be made to fit the data, thus indicating that the rheology of the asthenosphere is nonlinear. 
TABLE 2. Upper Mantle Parameters Inferred From the Rat Island Aftershock Migration Patterns

\begin{tabular}{lccc}
\hline & $n=2$ & $n=4$ & $n=6$ \\
\hline $\begin{array}{l}\text { Observed stress propagation coefficient } \kappa,{ }^{*} \\
\text { cgs units }\end{array}$ & $8 \times 10^{18 \pm 0.6}$ & $4 \times 10^{44 \pm 07}$ & $3 \times 10^{70 \pm 1}$ \\
$\begin{array}{l}\text { Calculated stress propagation coefficient } k,{ }^{\dagger} \\
\quad \text { cgs units }\end{array}$ & $1.3 \times 10^{20}$ & $1.6 \times 10^{42}$ & $2 \times 10^{84}$ \\
$\begin{array}{l}\text { Critical stress } \sigma_{x}{ }^{c},{ }^{*} \text { bars } \\
\text { Distance to which influence of earthquake } \\
\text { extends } x_{11 \mathrm{mut}},{ }^{*} \mathrm{~km}\end{array}$ & $11 \pm 2$ & $11 \pm 2$ & $11 \pm 2$ \\
\hline
\end{tabular}

*Data used were $x_{m}=175 \pm 25 \mathrm{~km}, t_{0}=2600 \pm 500$ days, $u_{0}=2.5 \mathrm{~m}, \tau=25 \mathrm{yr}$, and $E=1.6 \times 10^{\circ} \mathrm{bar}$. † We have assumed $h_{1}=70 \mathrm{~km}, h_{2}=50 \mathrm{~km}$, and $E=1.6 \times 10^{6}$ bar. $A$ is calculated from Kohlstedt and Goetze [1974] where we put $\dot{\epsilon}=10^{-9} \mathrm{~s}^{-1}$ for $\sigma=100$ bar at $1300^{\circ} \mathrm{C}$. (No correction for the pressure dependence of $A$ is included; such a correction would require somewhat higher temperatures to give the above results.)

quakes extends before the stresses drop to their average values and blend nonlinearly with the effects of previous earthquakes is calculated from (25). Table 2 shows this distance to be of the order of $300 \mathrm{~km}$, whatever $n$ we might choose.

We thus see that the effects of individual earthquakes are sharply limited to the margins of the lithospheric plate. Further than about $300 \mathrm{~km}$ from the plate margin the motion is dominated by the long-term average effect of many earthquakes (this conclusion was also reached by Bott and Dean [1973] on the basis of their $n=1$ model). Only a steady motion of the plate boundary can propagate stress and displacement fields rapidly into the plate interior. Equation (27), coupled with values of $k$ in Table 2 , shows that a steady motion of the plate boundary of $6 \mathrm{~cm} / \mathrm{yr}$ is propagated $5000 \mathrm{~km}$ into the interior of the plate in about $10^{4} \mathrm{yr}$, as opposed to $3 \times 10^{8} \mathrm{yr}$ for the influence of a single $u_{0}=10 \mathrm{~m}$ earthquake to propagate the same distance.

Note that the present work on stress propagation does not allow a decison to be made about the driving mechanism of plate tectonics. The predicted aftershock migration patterns are the same, independent of whether plates are pushed, pulled, or carried along by currents in the asthenosphere. Such a decision could be made only if it were possible to observe the manner in which the stress patterns from an individual earthquake merge with the average stress field, $300 \mathrm{~km}$ or so from the edge of the plate. Such an observation would give us information on the sign of the average shear stress in the asthenosphere and thus differentiate between theories of plate pushing or pulling and plate carrying. Unfortunately, this sort of observation seems so far beyond our present capability that it must remain a hypothetical possibility for some time to come.

We have arrived at a picture of stress propagation in the upper mantle which is consistent with both observation of aftershock migration patterns and laboratory measurements of high-temperature creep. We have found strong evidence that the rheology of the asthenosphere is nonlinear. Although much detailed work remains to be done, both on the observational side and in theoretical refinements, we believe that we have gained some insight into the nature of stress propagation in the upper mantle.

\section{A pPendix: Stress Propagation Over a Thick ASTHENOSPHERE}

The propagation of stress and displacement fields in an elastic lithosphere overlying a thin non-Newtonian asthenosphere was discussed in the main body of the paper. In this appendix we shall investigate the extent to which the above results must be modified in the case of a thick asthenosphere. The solution of the thick asthenosphere problem requires extensive numerical work except in the Newtonian, $n=1$ case. We shall thus concentrate on the $n=1$ solution and show that even for an infinitely thick asthenosphere (i.e., a viscous half space) the resulting stress pattern differs from the thin asthenosphere solution by no more than a factor of 2 . The stress propagation pattern is thus quite insensitive to the thickness of the asthenosphere for $n=1$. It seems plausible that this insensitivity should hold for the $n>1$ solutions as well, although this cannot be checked without detailed numerical computations. We shall see, in particular, that the corrections induced by a finite thickness asthenosphere are in the wrong direction to explain the observed aftershock patterns in terms of an $n=1$ asthenosphere.

We begin the thick asthenosphere solution by considering the relation between horizontal velocity $\dot{u}_{x}$ and shear stress $\sigma_{x z}$ at the top of a viscous slab with viscosity $\eta$ and thickness $h_{2}$. We first obtain the solution for a harmonic velocity field

$$
\dot{u}_{x}\left(k, h_{1}, t\right)=\dot{u}_{0}(k, t) e^{i k x}
$$

The boundary conditions are zero normal stress at the top of the asthenosphere, $\sigma_{z z}\left(h_{1}\right)=0$ (we have subtracted the lithostatic pressure field), and a rigid lower boundary at $z=h_{1}+$ $h_{2}$. The harmonic problem can be solved by standard techniques [Ramberg, 1967] using the stream friction $\psi(x, z)$. The general solution for a harmonically loaded viscous slab is

$$
\psi(x, z)=\left(A e^{k z}+B e^{-k z}+C z e^{k z}+D z e^{-k z}\right) e^{i k x}
$$

where $A, B, C$, and $D$ are constants determined by the boundary conditions. The velocities $\dot{u}_{x}$ and $\dot{u}_{z}$ are defined in terms of $\psi(x, z)$ :

$$
\begin{gathered}
\dot{u}_{x}(x, z)=\frac{\partial \psi}{\partial z}(x, z) \\
\dot{u}_{z}(x, z)=-\frac{\partial \psi}{\partial x}(x, z)
\end{gathered}
$$

The shear stress $\sigma_{x z}$ is given by $\sigma_{x z}=2 \eta \dot{\epsilon}_{x z}$; hence

$$
\sigma_{x z}(x, z)=\eta\left(\frac{\partial^{2} \psi(x, z)}{\partial z^{2}}-\frac{\partial^{2} \psi(x, z)}{\partial x^{2}}\right)
$$

The other stresses $\sigma_{x x}$ and $\sigma_{z z}$ may be found in a similar fashion [Ramberg, 1967].

Determination of $A, B, C$, and $D$ by use of the boundary conditions specified above yields a full solution for which the velocity and shear stress at the top of the asthenosphere are related by 


$$
\sigma_{x z}\left(x, h_{1}\right)=2 \eta k\left\{\frac{\left(k h_{2}\right)^{2}+\cosh ^{2}\left(k h_{2}\right)}{k h_{2}+\frac{1}{2} \sinh \left(2 k h_{2}\right)}\right\} \dot{u}_{x}\left(x, h_{1}\right)
$$

We proceed as in the section on fundamental equations in part I of the paper to balance this shear stress $\sigma_{x z}$ at the top of the asthenosphere (which thus acts on the base of the lithosphere) against the forces due to compression or tension of the lithosphere. Assuming a thin lithosphere (which is not essential but leads to a simpler argument), (2) yields

$$
\sigma_{x z}\left(x, h_{1}\right)=h_{1} E \frac{\partial^{2} u_{x}}{\partial x^{2}}=-h_{1} E k^{2} u_{x}\left(x, h_{1}\right)
$$

for a harmonic displacement at the lower boundary of the lithosphere. Equating (A5) and (A6), we obtain an equation for the displacement $u_{x}\left(k, h_{1}, t\right) e^{i k x}$ at the top of the asthenosphere for a harmonic displacement of wave number $k$ :

$$
\frac{d u_{x}}{d t}\left(k, h_{1}, t\right)=-\frac{h_{1} E}{2 \eta}\left[\frac{\left(k h_{2}\right)^{2}+\cosh ^{2}\left(k h_{2}\right)}{k h_{2}+\frac{1}{2} \sinh \left(2 k h_{2}\right)}\right] k u_{x}\left(k, h_{1}, t\right)
$$

This equation can immediately be integrated to yield

$$
\begin{aligned}
& u_{x}\left(k, h_{1}, t\right)=u\left(k, h_{1}, 0\right) \\
& \cdot \exp \left\{-\frac{h_{1} E}{2 \eta}\left[\frac{\left(k h_{2}\right)^{2}+\cosh ^{2}\left(k h_{2}\right)}{k h_{2}+\frac{1}{2} \sinh \left(2 h k_{2}\right)}\right] t\right\}
\end{aligned}
$$

This expression becomes much simpler in the limits $h_{2} \rightarrow 0$ or $h_{2} \rightarrow \infty$ :

$u_{x}\left(k, h_{1}, t\right)=u_{x}\left(k, h_{1}, 0\right) \exp \left\{-\frac{h_{1} h_{2} E}{\eta} k^{2} t\right\} \quad h_{2} \rightarrow 0$

$$
u_{x}\left(k, h_{1}, t\right)=u_{x}\left(k, h_{1}, 0\right) \exp \left\{-\frac{h_{1} E}{2 \eta}|k| t\right\} \quad h_{2} \rightarrow \infty
$$

These equations show how an individual Fourier component of wave number $k$ will relax in the two extreme cases of a thin asthenosphere $\left(h_{2} \rightarrow 0\right)$ and a thick asthenosphere $\left(h_{2} \rightarrow \infty\right)$. To describe the relaxation of a step function displacement at the edge of a plate, we determine the Fourier transform of the displacement at $t=0$, then let each component relax according to $(\mathrm{A} 9 a)$ or $(\mathrm{A} 9 b)$. If the edge of the plate is initially displaced a distance $u_{0}$, then the Fourier transform is

$$
u_{x}\left(k, h_{1}, 0\right)=\left(\frac{2}{\pi}\right)^{1 / 2} \frac{i u_{0}}{k+i \epsilon}
$$

where $\epsilon$ is an arbitrarily small positive number. Thus

$$
u_{x}(x, t)=\frac{1}{(2 \pi)^{1 / 2}} \int_{-\infty}^{\infty} u_{x}\left(k, h_{1}, t\right) e^{i k x} d k
$$

using (A9a) and (A9b)

$$
\begin{aligned}
& u_{x}(x, t)=\frac{i u_{0}}{\pi} \int_{-\infty}^{\infty} \frac{\exp \left[-\left(h_{1} h_{2} E / \eta\right) k^{2} t\right] \exp (i k x)}{k+i \epsilon} d k \\
& u_{x}(x, t)=\frac{i u_{0}}{\pi} \int_{-\infty}^{\infty} \frac{\exp \left[-\left(h_{1} E / 2 \eta\right)|k| t\right] \exp (i k x)}{k+i \epsilon} d k
\end{aligned}
$$

Integration of (A12a) yields (13) of the paper; this is the correct thin asthenosphere result. Integration of (A12b) yields the thick asthenosphere result

$$
u_{x}(x, t)=u_{0}\left[1-\frac{2}{\pi} \tan ^{-1}\left(\frac{2 \eta x}{h_{1} E t}\right)\right]
$$

Note that this equation satisfies the usual conditions $u_{x}(x, t)=$ $u_{0}$ at $x=0$ for all $t$ and $u_{x}(x, t)=0$ at all $x \neq 0$ for $t=0$. Substitution of (A8) into (Al1) will yield an expression for $u_{x}(x, t)$ which is valid for finite $h_{2}$, being a function of the parameter $h_{2} / h_{1}$. Such solutions are bounded by the extreme solutions for $h_{2} \rightarrow 0$ and $h_{2} \rightarrow \infty$, so that by comparing these extremes, we can estimate the possible variation in the model's results due to a change in the thickness of the asthenosphere.

Proceeding as in the section on step function initial displacement in part $\mathrm{I}$, we find that the stress $\sigma_{x}$ in the plate in the $h_{2}$ $\rightarrow \infty$ limit is given by

$$
\frac{\sigma_{x}}{E}=\frac{2}{\pi} \frac{2 \eta u_{0}}{h_{1} E t}\left[1+\left(\frac{2 \eta x}{h_{1} E t}\right)^{2}\right]^{-1}
$$

from which the distance of a stress contour (where $\sigma_{x}(x, t)=$ $\sigma_{c}$ ) from the edge of the plate can be found as a function of time:

$$
\frac{x}{x_{m}}=2\left(\frac{t}{t_{0}}\right)^{1 / 2}\left(1-\frac{t_{0}}{t}\right)^{1 / 2}
$$

where $x_{m}$ is the maximum distance attained by the contour (which occurs at time $t_{m}=t_{0} / 2$ ). The contour then retreats toward the plate edge, as described in the paper, and finally attains it at time $t_{0}$. If $x_{m}$ and $t_{m}$ are known (by measurement), then the viscosity $\eta$ and value of the stress $\sigma_{c}$ can be derived from

$$
\begin{array}{rlrl}
\eta & =\frac{h_{1} E}{4} \frac{t_{0}}{x_{m}} & h_{2} \rightarrow \infty \\
\frac{\sigma_{c}}{E}=\frac{1}{\pi} \frac{u_{0}}{x_{m}} & h_{2} \rightarrow \infty
\end{array}
$$

These expressions can be compared with (20) and (21) in the paper, which yield $(n=1)$

$$
\begin{array}{rlrl}
\eta & =\frac{2 h_{1} h_{2} E}{e x_{m}} \frac{t_{0}}{x_{m}} & h_{2} \rightarrow 0 \\
\frac{\sigma_{c}}{E}=\left(\frac{2}{e \pi}\right)^{1 / 2} \frac{u_{0}}{x_{m}} & h_{2} \rightarrow 0
\end{array}
$$

Note that (A17) and (A19) differ only by $30 \%$, while the ratio of (A16) and (A18) is about $x_{m} / 3 h_{2}$. If $x_{m} \simeq 200 \mathrm{~km}$ and $h_{2} \simeq$ $50 \mathrm{~km}$, then we only have a $30 \%$ discrepancy here also. Moreover, Figure 4 shows that the position of a stress contour versus time is very nearly the same for the $h_{2} \rightarrow \infty$ and $h_{2} \rightarrow 0$ limits.

The conclusion of this analysis is that for $n=1$, the stress propagation pattern due to a step function displacement of the edge of a plate is quite insensitive to the thickness of the viscous layer underlying the plate. The thin slab approximation is thus justified for $n=1$. This approximation has yet to be justified for $n>1$. However, we believe that it is plausible that our results for $n>1$ will not be greatly altered in the case of an asthenosphere whose thickness is some fraction of $x_{m}$.

Acknowledgments. I would like to thank Hiroo Kanamori for his aid in the selection of the Rat Island earthquake and his willingness to listen to my half-formed ideas. Gordon Stewart also aided me in collecting aftershock data. Contribution 2638 of the Division of Geological and Planetary Sciences, California Institute of Technology, Pasadena, California 91125.

\section{REFERENCES}

Anderson, D. L., Accelerated plate tectonics, Science, 187, 1077-1079, 1975.

Bott, M. H. P., and D. S. Dean, Stress diffusion from plate boundaries, Nature, 243, 339-341, 1973. 
Bullen, K. E., Introduction to the Theory of Seismology, 3rd ed., p. 233 Cambridge University Press, New York, 1965.

Crank, J., The Mathematics of Diffusion, p. 31, Oxford University Press, New York, 1956.

Elsasser, W. M., Convection and stress propagation in the upper mantle, in The Application of Madern Physics to the Earth and Planetary Interiors, edited by S. K. Runcorn, pp. 223-246, John Wiley, New York, 1967.

Gutenberg, B., and C. F. Richter, Seismicity of the Earth, Princeton University Press, Princeton, N. J., 1954.

Jordan, J. N., J. F. Lander, and R. A. Black, Aftershocks of the 4 February 1965 Rat Island earthquake, Science, 148, 1323-1325, 1965.

Kohlstedt, D. L., and C. Goetze, Low-stress, high-temperature creep in olivine single crystals, J. Geophys. Res., 79, 2045-2051, 1974.

Le Pichon, X., Sea floor spreading and continental drift, J. Geophys. Res. 73, 3661-3697, 1968.

Molnar, P., and M. Wyss, Moments, source dimensions and stress drops of shallow-focus earthquakes in the Tonga-Kermadec arc, Phys. Earth Planet. Interiors, 6, 263-278, 1972.

Post, R. L., and D. T. Griggs, The earth's mantle: Evidence of nonNewtonian flow, Science, 181. 1242-1244, 1973.

Ramberg, H., Gravity. Deformation and the Earth's Crust, p. 179, Academic, New York, 1967.

Stauder, W., Mechanism of the Rat Island earthquake sequence of February 4, 1965, with relation to island arrcs and sea floor spreading, J. Geophys. Res., 73, 3847-3858, 1968.

Weertman, J., and J. R. Weertman, High temperature creep of rock and mantle viscosity, Annu. Rev. Earth Planet. Sci., 3, 293-315, 1975.

Wu, F. T., and H. Kanamori, Source mechanism of February 4, 1965, Rat Island earthquake, J. Geophys. Res., 78, 6082-6092, 1973.

(Received July 3, 1975;

revised June 16, 1976;

accepted June 17, 1976.) 